\title{
Sound propagation in light-modulated carbon nanosponge suspensions
}

\author{
W. Zhou, ${ }^{1}$ R. P. Tiwari, ${ }^{1}$ R. Annamalai, ${ }^{2}$ R. Sooryakumar, ${ }^{1}$ V. Subramaniam, ${ }^{2}$ and D. Stroud ${ }^{1}$ \\ ${ }^{1}$ Department of Physics, The Ohio State University, Columbus, Ohio 43210, USA \\ ${ }^{2}$ Department of Mechanical Engineering, The Ohio State University, Columbus, Ohio 43210, USA \\ (Received 29 May 2008; revised manuscript received 22 January 2009; published 18 March 2009)
}

\begin{abstract}
Single-walled carbon nanotube bundles dispersed in a highly polar fluid are found to agglomerate into a porous structure when exposed to low levels of laser radiation. The phototunable nanoscale porous structures provide an unusual way to control the acoustic properties of the suspension. Despite the high sound speed of the nanotubes, the measured speed of longitudinal-acoustic waves in the suspension decreases sharply with increasing bundle concentration. Two possible explanations for this reduction in sound speed are considered. One is simply that the sound speed decreases because of fluid heat induced by laser light absorption by the carbon nanotubes. The second is that this decrease results from the smaller sound velocity of fluid confined in a porous medium. Using a simplified description of convective heat transport, we estimate that the increase in temperature is too small to account for the observed decrease in sound velocity. To test the second possible explanation, we calculate the sound velocity in a porous medium, using a self-consistent effective-medium approximation. The results of this calculation agree qualitatively with experiment. In this case, the observed sound wave would be the analog of the slow compressional mode of porous solids at a structural length scale of order of $100 \mathrm{~nm}$.
\end{abstract}

DOI: 10.1103/PhysRevB.79.104204

PACS number(s): 62.30.+d, 78.35.+c, 61.43.Gt, 82.70.Dd

\section{INTRODUCTION}

Materials with nanometer-sized pores, often referred to as nanosponges, are finding numerous applications. For example, they can serve as contaminant absorbers, ${ }^{1}$ carriers for drug delivery, ${ }^{2}$ catalysts for oxygen reduction, ${ }^{3}$ and templates for producing defect-free ultralow- $k$ dielectrics. ${ }^{4}$ Typically, the sol-gel method is used to make these nanosponges, in which a solution containing nanoparticles in suspension is polymerized to form a gel. ${ }^{5}$

In this study, we report on the creation of nanosponges made by dispersing networks of single-walled carbon nanotube (SWCNT) bundles in a polar fluid and exposing the suspension to low-level laser radiation $\left(\sim 2.5 \mathrm{~kW} / \mathrm{cm}^{2}\right)$. Remarkably, exposure to laser light leads to agglomeration of the dispersed bundles into a nanoporous structure whose average pore dimensions and volume fraction within the focal volume are tunable by light. Since the physical scaling lengths associated with such fluids-such as the wavelength of sound-are typically larger than the dimensions of the nanopores, these suspensions offer excellent opportunities to investigate the propagation of acoustic waves through inhomogeneous media.

Sound propagation in liquid-solid mixtures has been studied extensively. For example, Biot ${ }^{6}$ proposed that, in a biconnected liquid-solid mixture, there are typically three sound modes: a fast compressional mode, a shear mode, and a slow compressional mode. This work has been followed by many additional theoretical and experimental studies (e.g., Refs. 7-11) of wave propagation in liquid-solid composites. These works consider composites as diverse as colloidal suspensions, ${ }^{8}$ superfluid helium in aerogel, ${ }^{9}$ and watersaturated sand. ${ }^{10}$

Here we experimentally investigate propagation of sound in liquid-solid composites but in a different regime where the structural length scale is $\sim 100 \mathrm{~nm}$. Such suspensions are of interest scientifically because of the sensitivity of sound propagation to connectivity and nanoscale structure, and technologically because of possible opportunities for control of the spongy nanobundle structure with light and improved heat-management applications. ${ }^{12}$ We study these acoustic properties by Brillouin light scattering. ${ }^{13,14}$ Despite the stiffness of the SWCNTs, our measurements reveal an unexpected softening of the sound velocity of the suspension with increasing initial weight fraction of the bundles. We complement our experimental study with a simple effective-medium model for sound propagation in these media.

\section{SAMPLE PREPARATION AND EXPERIMENTAL RESULTS}

We produce solutions containing dispersed bundles of single-walled carbon nanotubes by first adding $1 \mathrm{mg}$ of the carbon material (CarboLex, AP grade, as produced with $50 \%-70 \%$ purity) to $100 \mathrm{~mL}$ of $\mathrm{N}, \mathrm{N}$-dimethylformamide (DMF). Upon sonication for $45 \mathrm{~min}$, the carbon material becomes uniformly dispersed in the DMF solution. This solution is then diluted further with DMF to obtain concentrations ranging from $5 \times 10^{-5}$ to $9 \times 10^{-4} \mathrm{~g} / \mathrm{L}$, and sonicated again until the solute is evenly dispersed.

Previous work ${ }^{15}$ has shown that these sonicated mixtures contain bundles of $1.4 \mathrm{~nm}$ diameter SWCNTs. The bundles are $\sim 25 \mathrm{~nm}$ in diameter and several microns long [Fig. 1(a)]. Moreover, the sonication process produces both positively and negatively charged bundles. The positively charged bundles are solvated by the highly polar DMF molecules so that only the negatively charged bundles are free to migrate. ${ }^{15}$ The fact that the migratory bundles are negatively charged becomes evident when a positive bias is applied to the solution. ${ }^{15}$

Figure 1(b) shows a transmission electron microscope (TEM) image of the bundles after exposure to $514.5 \mathrm{~nm}$ laser 

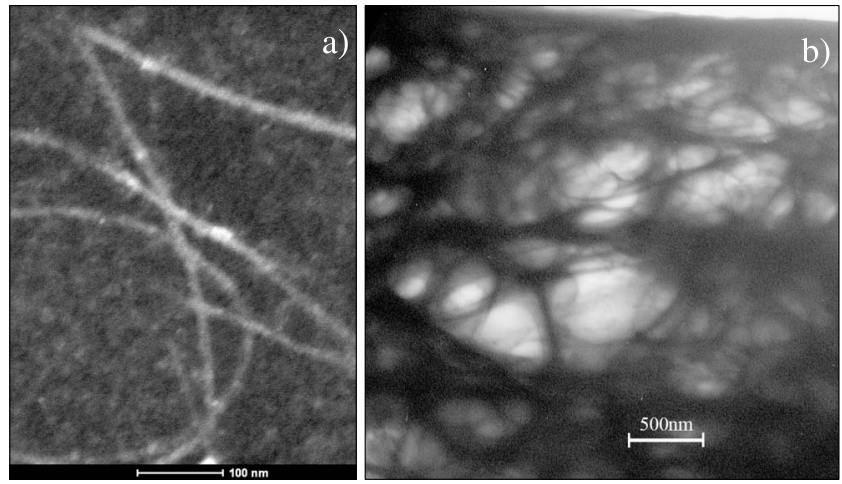

FIG. 1. (a) TEM image of SWCNT. The images were from specimens obtained by drying a sonicated DMR-SWCNT bundle suspended on a grid. (b) TEM image of light-induced agglomerated bundles. The clear regions are pores while the dark regions are the SWCNT bundles.

radiation. There are several possible mechanisms underlying the observed light-driven agglomeration. First, the charge on the bundles might be modified by electron emission due to the laser electric field. Such field emission from SWCNTs is well known. ${ }^{16,17}$ In fact, enhancement factors exceeding $10^{4}$ that reflect the diameter, length, and packing density of SWCNT bundles have been reported. ${ }^{17}$ This allows applied fields of $10^{5}-10^{6} \mathrm{~V} / \mathrm{m}$ to initiate electron emission from the bundles. These relatively low turn-on fields are, in fact, easily realized by the laser electric field for the power densities used in this study. Second, the charge on the bundles could be modified by localized laser-induced heating. ${ }^{18}$ These mechanisms could initiate nanobundle agglomeration by disturbing local charge neutrality. Energy transfer from the nanobundles may also heat the DMF near the focal volume, leading to thermally driven convective bulk fluid motion and to further growth of the agglomerates as they sweep through the fluid. We have observed such bulk motion of the fluid, and display the resulting larger spongelike structures in the TEM image of Fig. 1(b). The pore dimensions are mostly less than $500 \mathrm{~nm}$, i.e., of the order of the wavelength of sound probed in this study. Thus, one expects the sound velocity in the suspension to differ substantially from that of the pure liquid, as indeed the measurements show.

The acoustic modes in the suspension are detected by Brillouin scattering, using laser excitation at wavelength $\lambda$ $=514.5 \mathrm{~nm}$. The incident power was maintained at $\sim 50 \mathrm{~mW}$, focused to a spot size of $\sim 50 \mu \mathrm{m}$ in diameter, and the backscattered spectra from freshly sonicated suspensions were recorded. The frequency of the longitudinal phonon is measured from the Brillouin doublet at a fixed wave vector $q=(4 \pi n / \lambda) \sin \theta / 2$, where $n$ is taken to be the refractive index of the liquid, and $\theta=180^{\circ}$ in backscattering. Now the index of refraction of pure SWCNT in the visible is estimated to be approximately two, as extrapolated from measurements of SWCNTs at lower frequencies, and of highly porous SWCNT films. ${ }^{19,20}$ Since this is larger than $n_{\mathrm{DMF}}$, the index of refraction of the suspension is greater than that of pure DMF. If $c_{\text {expt }}$ were obtained using the value of the suspension index, the speed would thus be even lower than that shown in Fig. 3.

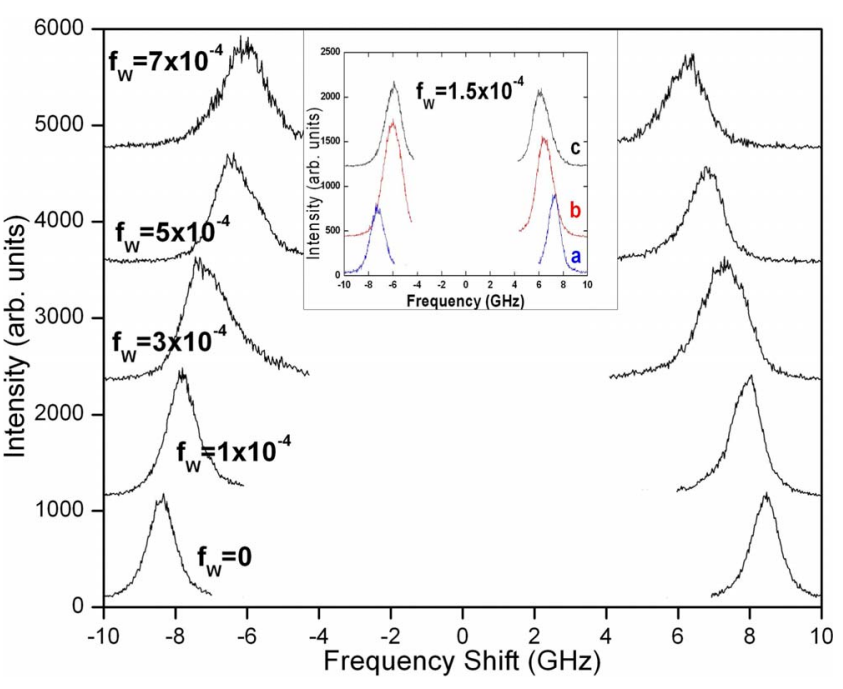

FIG. 2. (Color online) Frequency shift of the backscattered Brillouin doublet (in $\mathrm{GHz}$ ) as a function of the initial weight fraction $f_{W}$ of CNT bundles in the suspension. All Brillouin spectra were recorded for two minutes. In all cases in the main figure with $f_{W}$ $\neq 0$, the Brillouin spectra were measured immediately after sonication. The inset shows the Brillouin doublet at $f_{W}=1.5 \times 10^{-4}$, recorded (a) after sonication for $1 / 2 \mathrm{~h}$, (b) after simultaneous sonication and exposure to $514.5 \mathrm{~nm}$ laser radiation for $1 / 2 \mathrm{~h}$, and (c) after ceasing sonication but continuing to expose the sample to $514.5 \mathrm{~nm}$ laser illumination for $1 / 2 \mathrm{~h}$.

The mode frequencies were measured for different initial weight fractions $f_{W}$ of the nanotube bundles, and their phase velocities $(v=\omega / q)$ determined from the frequencies of the Brillouin peaks. The weight fraction of the nanobundles within the focal volume of the incident probe-laser beam is time dependent and greater than $f_{W}$ because of agglomeration. Therefore, most measurements are recorded not only immediately after sonication but also after exposing the suspension to laser radiation for specified time periods.

Figure 2 shows a sequence of Brillouin spectra recorded for two minutes from the DMF-SWCNT suspension with different $f_{W}$. For all $f_{W}$, we observe only one mode, as evident from the single Stokes/anti-Stokes Brillouin doublet. With increasing $f_{W}$, the Brillouin peaks clearly shift to lower frequencies and become slightly asymmetric toward the lowfrequency side. Given the structural rigidity of the nanotubes and their very high sound velocity (more than 100 times that of pure DMF), the observed decrease in frequency is counterintuitive. Moreover, the relatively large (25\%) decrease in the frequency (and therefore the sound velocity) of the suspension with modest $f_{W}\left(\sim 7 \times 10^{-4}\right)$ is surprising. The variation in the speed of the acoustic mode with $f_{W}$ is summarized in Fig. 3.

To understand the large decrease in acoustic velocities with increasing $f_{W}$, additional Brillouin experiments were performed to identify contributions to self-agglomeration from light exposure, and the countereffects due to sonication. Sonication leads to the systematic breakup of the nanosponges and eventually to the creation of isolated nanotube bundles. The inset of Fig. 2 illustrates this effect for $f_{W}$ $=1.5 \times 10^{-5}$. Initially, the suspension was sonicated for $1 / 2 \mathrm{~h}$ 


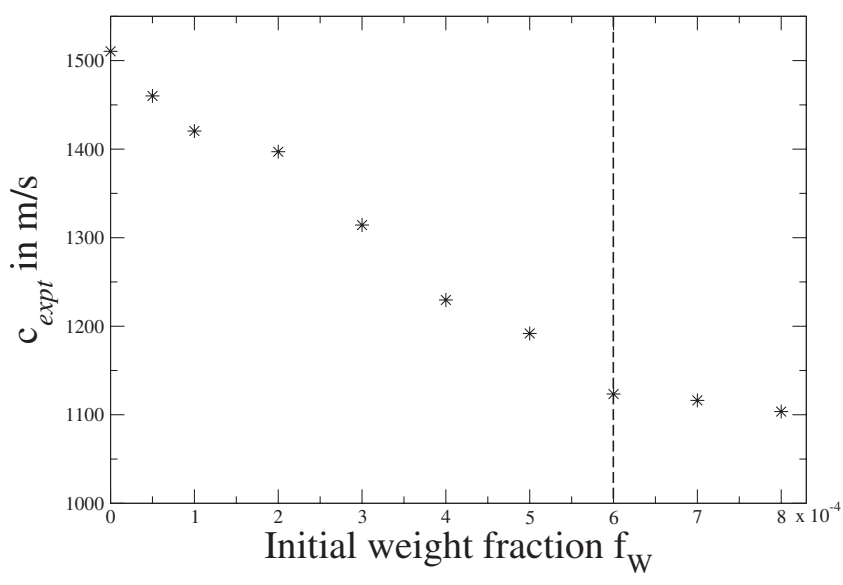

FIG. 3. Sound speed $c_{\text {expt }}$ in $\mathrm{m} / \mathrm{s}$, as extracted from the peak positions in the Brillouin doublet, as a function of the initial weight fraction of SWCNT bundles in the suspension. The dashed line denotes the value of $f_{W}$ beyond which $c_{\text {expt }}$ changes little. Note that $f_{W}$ is $10^{2}-10^{3}$ times smaller than the weight fraction in the focal volume.

without laser exposure to ensure that no agglomeration occurred immediately prior to the measurement. Next, a Brillouin spectrum was acquired (while continuing the sonication) with $514.5 \mathrm{~nm}$ laser light. This measurement yielded a longitudinal mode at a frequency of $7.1 \mathrm{GHz}$ [curve (a) of Fig. 2]. To distinguish the competing effects of laser-driven self-agglomeration and fragmentation from sonication, illumination of the suspension with $514.5 \mathrm{~nm}$ laser light was continued for an additional $1 / 2 \mathrm{~h}$, together with ongoing sonication. At the end of this period, a Brillouin spectrum was recorded, which showed that the mode frequency of the suspension had dropped from 7.1 to $5.9 \mathrm{GHz}$ [curve (b) of Fig. 2]. This decrease is consistent with our finding that exposure to light causes agglomeration of the carbon nanobundles, overcoming the breakup due to sonication, and yielding a corresponding decrease in the sound velocity. In order to confirm that the decrease in the sound velocity is primarily due to the laser-driven agglomeration, we stopped the sonication but continued the laser exposure for another $1 / 2$ h. Following this, the mode frequency was measured again, and observed to decrease further to $5.8 \mathrm{GHz}$ [curve (c) of Fig. 2].

Figure 3 summarizes the variation in the measured sound speed $c_{\text {expt }}$ with $f_{W}$. Beginning with $f_{W} \sim 5 \times 10^{-5}$, we found that the frequency, and thus the sound velocity, of the suspension decreases monotonically from the value of $8.4 \mathrm{GHz}$ in the pure DMF solvent. The frequency continues to decrease until $f_{W} \sim 6 \times 10^{-4}$, beyond which the mode frequency stabilizes and appears unaffected by further increases in SWCNT concentration.

\section{ANALYSIS OF THE EXPERIMENTAL RESULTS}

\section{A. Effects of heating}

We now turn to the interpretation of these sound velocity measurements. One possibility to consider is that the observed drop in the sound velocity is due to local heating of the DMF liquid resulting from absorption of laser energy by the SWCNTs. To check this possibility, we have, first of all, directly measured the change in sound velocity of pure DMF as a function of temperature, by measuring its temperaturedependent Brillouin spectrum from $T=30{ }^{\circ} \mathrm{C}$ to $T=105^{\circ} \mathrm{C}$. We find that the frequency of the longitudinal mode drops smoothly with increasing temperature from about $8.1 \mathrm{GHz}$ at $30{ }^{\circ} \mathrm{C}$ to $6.15 \mathrm{GHz}$, at $105{ }^{\circ} \mathrm{C}$, a decrease of about $24 \%$. If the index of refraction is, as seems likely, very weakly temperature dependent, this measurement would indicate a decrease in the speed of the longitudinal mode also of about $24 \%$ over this temperature range. While this is comparable to the decrease in sound speed of the suspension which we observe, the actual increase in the temperature of the suspension is expected to be only a few kelvin, as we now argue.

Heating of the DMF occurs indirectly. The laser energy is first absorbed by the SWCNT bundles and dissipated to the surrounding fluid. This results in a localized heated region within the fluid where the temperature is higher than that of the surrounding cooler DMF, and causes a flow driven by natural convection. An estimate of the temperature rise of the laser-irradiated region may be obtained by examining the momentum conservation equation for a fluid flowing under the influence of natural convection. Using the Boussinesq approximation, ${ }^{21}$ the body force in free convection may be linearized. This is done by relating the density difference to a temperature difference via the volumetric thermal-expansion coefficient, $\beta$. Then, comparing the inertia term and this linearized body force term in the streamwise momentum equation yields ${ }^{21}$

$$
u_{\max }^{2} \sim 2 g L \beta\left(T-T_{\infty}\right),
$$

where $u_{\max }$ is the maximum induced velocity of the flow (which we measured by tracking a particulate), $L$ is a characteristic length scale, which is taken to be the spot size of the laser, $\beta=-\rho^{-1}(\partial \rho / \partial T)_{P}$ is the volume thermal-expansion coefficient, $T_{\infty}$ is the surrounding fluid temperature (taken as $300 \mathrm{~K}), T$ is the temperature in the laser spot, and $g$ is the acceleration of gravity.

The density of DMF is $925 \mathrm{~kg} / \mathrm{m}^{3}$ at $318 \mathrm{~K}$ and $961 \mathrm{~kg} / \mathrm{m}^{3}$ at $278 \mathrm{~K}$. A straight-line fit to this data gives $\rho_{\mathrm{DMF}}(T)=-0.917 T+1217$, where $T$ is in kelvin and $\rho$ in $\mathrm{kg} / \mathrm{m}^{3}$. In order to estimate $u_{\max }$, we observed and recorded the positions of the agglomerated particles in suspension as a function of time. From these observations, we estimate $u_{\max }$ to be no greater than $2 \times 10^{-3} \mathrm{~m} / \mathrm{s}$. If we take the laser spot size to be $5 \times 10^{-5} \mathrm{~m}$, we then obtain $T-T_{\infty} \sim 4 \mathrm{~K}$. Thus, we estimate the localized heating of the DMF liquid to be less than $10 \mathrm{~K}$. According to the above measurements of the temperature-dependent sound velocity in DMF, this temperature change would produce only a $1 \%-2 \%$ change in the sound velocity, too small to account for the observed velocity change.

\section{B. Composite effect modeling of the sound velocity in the suspensions}

We are thus led to an alternative interpretation of these measurements by considering the sound velocity of the sus- 
pension created by the laser-induced agglomeration. To calculate this sound velocity, we use a simple effective-medium approximation (EMA). Several such approximations have been developed both for random composites of solid grains ${ }^{22}$ and for suspensions of solid particles in a liquid host. ${ }^{23}$ These approximations appear to be quite good for compressional modes, ${ }^{24}$ such as are studied here. We consider a suspension consisting of two components: a liquid (DMF; medium 1) and a solid (the nanotube bundles, medium 2), present in volume fractions $1-f_{V}$ and $f_{V}$, respectively. Since the aggregates shown in Fig. 1(b) appear macroscopically isotropic, we follow standard approaches to effective-medium theories, and treat the composite as if it were composed of small approximately spherical units, each of the same radius $a$, and randomly distributed in the composite. The liquid and solid are assumed to have compressional sound speeds $c_{\ell}$ and $c_{s}$ $\gg c_{\ell}$. Other more elaborate approaches are also feasible but we believe that they would give qualitatively similar results, as discussed further below. Within the liquid, the local pressure $p(\mathbf{r})$ at position $\mathbf{r}$ satisfies ${ }^{25} \nabla^{2} p(\mathbf{r})+\left(\omega^{2} / c_{\ell}^{2}\right) p(\mathbf{r})=0$. If the solid is taken to be infinitely stiff, the only necessary boundary condition ${ }^{25,26}$ is that the normal derivative $\partial p_{1} / \partial n$ $=0$ at the solid-liquid boundary.

In the EMA, the entire suspension is replaced by an effective medium whose speed of sound, $c_{\text {eff }}$, is determined by embedding each component particle in a self-consistently determined effective medium. The pressures in the effective medium and the constituent media all satisfy the appropriate Helmholtz equations. We impose the boundary condition mentioned above, and the self-consistency requirement that the forward scattering from the components embedded in the effective medium should vanish, on the average. ${ }^{27}$ This condition leads to a self-consistent equation which implicitly determines the one unknown quantity, the effective wave vector $k_{\text {eff }}$, as a function of $\omega, c_{\ell}$, and $f_{V}$ :

$$
\begin{aligned}
& \sum_{n=0}^{\infty}(2 n+1)\left[f_{V} \frac{j_{n}^{\prime}\left(k_{\mathrm{eff}} a\right)}{h_{n}^{(1),{ }^{\prime}}\left(k_{\mathrm{eff}} a\right)}+\left(1-f_{V}\right)\right. \\
& \left.\quad \times \frac{k_{\mathrm{eff}} j_{n}^{\prime}\left(k_{\mathrm{eff}} a\right) j_{n}\left(k_{\ell} a\right)-k_{\ell} j_{n}\left(k_{\mathrm{eff}} a\right) j_{n}^{\prime}\left(k_{\ell} a\right)}{k_{\mathrm{eff}} h_{n}^{(1),{ }^{\prime}}\left(k_{\mathrm{eff}} a\right) j_{n}\left(k_{\ell} a\right)-k_{\ell} h_{n}^{(1)}\left(k_{\mathrm{eff}} a\right) j_{n}^{\prime}\left(k_{\ell} a\right)}\right]=0,
\end{aligned}
$$

where $j_{n}$ and $h_{n}^{(1)}$ denote the spherical Bessel function and the spherical Hankel function of the first kind, and the prime denotes a derivative with respect to the argument. A related approximation has been described in Ref. 23 except that the self-consistency condition in Ref. 23 involves embedding liquid-coated solid spheres in an effective medium.

We have solved Eq. (2) self-consistently for $c_{\text {eff }} / c_{\ell}$ as a function of $\omega a / c_{\ell}$. The results, shown in Fig. 4, depend on $\omega a / c_{\ell}$, particularly at higher $f_{V}$ where $c_{\text {eff }}$ is significantly smaller than $c_{\ell}$. The sum converges very rapidly, with only three terms $(n=0,1,2)$ sufficing for an accuracy of $0.1 \%$ or better in the regime $\omega a / c_{\text {eff }} \leq 1$ and $f_{V} \leq 1 / 2$. The results show that, as $f_{V}$ increases, $c_{\text {eff }}$ decreases. One interpretation of this result is that the presence of SWCNTs causes the sound wave propagating in the fluid to undergo more scattering, and hence, to have a lower sound velocity. As the

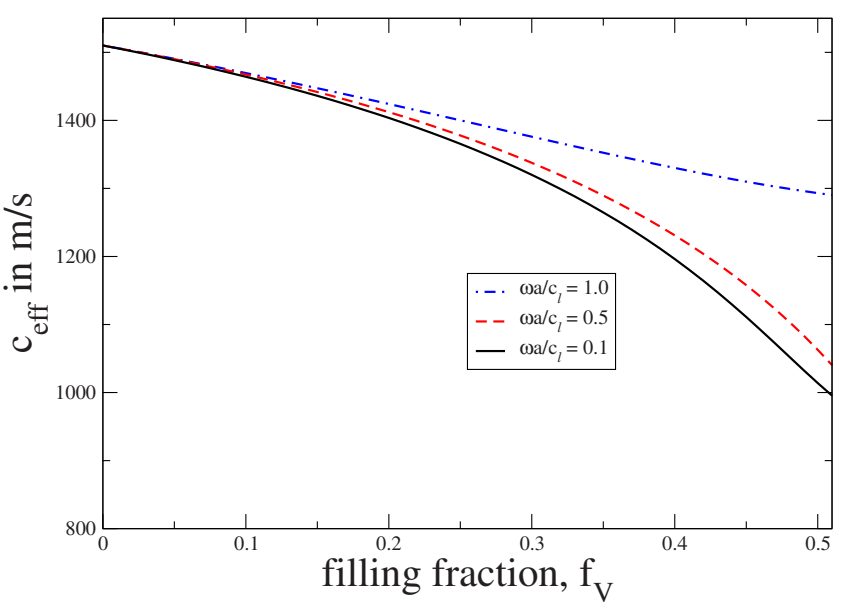

FIG. 4. (Color online) Effective sound velocity $c_{\text {eff }}$ versus volume fraction $f_{V}$ for the suspension of SWCNT nanobundles calculated using Eq. (2), assuming that the liquid is DMF, and considering three values of the parameter $\omega a / c_{\ell}$, where $f_{V}$ the volume fraction of the solid component.

concentration of SWCNTs increases, one would expect that this scattering would also increase and hence that the reduction in sound velocity would be greater. Thus, the reduction in the sound velocity with increasing $f_{V}$ seen in the EMA also seems intuitively reasonable.

Other theoretical studies have also obtained a decrease in sound velocity with increasing volume fraction of stiff material similar to what we find here. For example, a similar reduction has also been derived analytically for sound propagating in a fluid containing a periodic array of infinitely stiff cylinders, ${ }^{28}$ using a Green's-function-based multiplescattering approach. In addition, Berryman ${ }^{22}$ has derived a similar reduction in sound velocity for a dilute suspension $\left(f_{V} \ll 1\right)$ of a stiff solid in a liquid host. His result is $c_{\text {eff }} / c_{\ell}$ $=1 / \sqrt{\alpha}$, with $\alpha=(1 / 2)\left[1+1 /\left(1-f_{V}\right)\right]$. This same result can also be derived by considering the effective-medium approximation in the limit $\omega a / c_{\ell} \ll 1$ and $f_{V} \ll 1$. In this regime, Eq. (2) can be solved analytically, and it is found that only the $n=0$ and $n=1$ terms contribute. The result of the EMA in this limit is $c_{\text {eff }} / c_{\ell} \sim 1-f_{V} / 4$, which agrees, to first order in $f_{V}$, with Berryman's ${ }^{22}$ result.

Another interesting regime for the effective-medium approximation is that for which $1 / 3<f_{V}<2 / 3$. At these concentrations, the porous medium is expected to be bicontinuous in the EMA, ${ }^{29}$ i.e., both the liquid and the solid phases form a connected path extending throughout the sample. In this regime, the mode whose sound velocity we calculate is analogous to the slow compressional mode often discussed in porous solids. ${ }^{30}$ The frequency of this mode is expected to approach zero when the liquid phase ceases to have such a connected path extending throughout the sample. In the EMA, this limit occurs at $f_{V}=2 / 3$. Indeed, we find that $c_{\ell}$, in our calculation, approaches zero when $f_{V} \rightarrow 2 / 3$ and $\omega a / c_{\ell}$ $\ll 1$, as suggested by this interpretation.

We now discuss one feature of our results which may seem unrealistic for the carbon nanotube bundles. Namely, we have modeled the suspension as a random mixture of spheres of liquid and of very stiff solid. In reality, of course, 
each SWCNT bundles would be better described as a stiff cylinder. Thus, a more realistic modeling approach would be to treat the suspension as a mixture of randomly oriented cylinders representing the SWCNT bundles and other shapes (perhaps ellipsoids) representing the liquid, and then to carry out an effective-medium approximation to obtain the sound velocity of this mixture. However, it is quite difficult to execute an EMA for this complicated geometry. Therefore, as an illustration of the influence of geometry, we have also carried out a similar effective-medium approximation for a random distribution of parallel cylinders, calculating the sound velocity perpendicular to the cylinder axis. In this case, one embeds cylinders, rather than spheres, of very stiff material (representing the SWCNT bundles) and liquid in a self-consistently determined effective medium. The selfconsistency condition then involves cylindrical, rather than spherical, Bessel functions. The results from this type of modeling in the limit of very stiff SWCNT bundles are qualitatively similar to those shown in Fig. 4: the sound velocity again decreases with increasing SWCNT concentration, and a similar dependence on cylinder radius is obtained. The percolation threshold for $f_{V}$, above which there is no connected path for liquid through the sample, is now $f_{V}=1 / 2$ rather than $f_{V}=2 / 3$ obtained for spheres. We therefore conclude that the qualitative behavior seen in Fig. 4 is insensitive to the exact details of the model used to treat sound propagation in composites of SWCNT bundles and DMF. Of course, it would be desirable, although difficult, to make the present model more realistic by developing an effective-medium theory involving an orientational average over randomly oriented cylindrical SWCNT bundles but the general features of the behavior would not be expected to change greatly.

The experimental results may now be discussed in view of these calculations. In all the experiments, $f_{W} \sim 10^{-4}$, as is the corresponding initial volume fraction of the SWCNTs. ${ }^{31}$ However, once the laser beam is turned on, the SWCNT bundles are advected into the beam and there form aggregates whose weight (or volume) fraction is a factor of $10^{2}-10^{3}$ greater than $f_{W}$. Hence, the measurements should be compared to calculations for values of $f_{V}$ in the range of $0.2-0.4$. For these values of $f_{V}$, if we take $a$ to be the radius $(\sim 25 \mathrm{~nm})$ of one SWCNT bundle, we obtain $\omega a / c_{\ell} \sim 0.5$ and hence $c_{\text {eff }} / c_{\ell} \sim 0.8-0.9$, comparable to the measured $c_{\text {expt }} / c_{\ell}$. Another feature of the experimental results is that $c_{\text {expt }}$ saturates for $f_{W}>6 \times 10^{-4}$. According to our interpretation, this means that the volume fraction of SWCNTs in the focal volume ceases to increase for $f_{W}$ greater than this value. This saturation probably occurs because, for $f_{W}>6$ $\times 10^{-4}$, the size of the spongy nanostructure created by the laser-driven agglomeration exceeds the dimensions of the focal volume, setting an upper limit on $f_{V}$ within the focal volume and hence, a lower limit to the sound velocity.

\section{SUMMARY AND CONCLUSIONS}

In summary, we have carried out Brillouin scattering experiments on suspensions of SWCNT bundles in an organic solvent of DMF. Under laser illumination, the bundles agglomerate, leading to a substantial decrease in the measured sound velocity with increasing SWCNT concentration. This sound velocity is significantly lower than that of pure DMF, even though the SWCNT bundles have a much higher sound speed than the DMF. We have estimated the change in sound velocity due to local heating of the DMF. From estimates of the change in temperature due to this heating, combined with our measurements of the temperature-dependent sound velocity of pure DMF, we find that such heating is too small to account for the observed drop in sound velocity. However, the reduction in sound speed, as calculated using the EMA, is comparable to that seen in the experimentally studied suspension. We interpret the observed acoustic mode as the analog of the slow compressional mode in porous solids having a structural length scale of order of $100 \mathrm{~nm}$. We conclude that the reduction in sound velocity is due primarily to composite effects arising from agglomeration. Our experimental work should be useful in developing phototunable nanosponges, as well as creating different types of fluids with nanoscale inhomogeneities, in which the sound velocity can be systematically controlled using light.

\section{ACKNOWLEDGMENTS}

This work has been supported, in part, by NSF Grants No. DMR-0205521 and No. DMR-0413395, by the Center for Emergent Materials at the Ohio State University (an NSF MRSEC; Grant No. DMR 0820414), and by the Ohio State University Institute of Materials Research. We thank X. Xie for contributions to this work.
${ }^{1}$ M. Arkas, R. Allabash, T. Tsiourvas, E. M. Matteusch, and R. Perfler, Environ. Sci. Technol. 40, 2771 (2006).

${ }^{2}$ R. Cavalli, F. Trotta, and W. Tumiatti, J. Incl. Phenom. Macrocycl. Chem. 56, 209 (2006).

${ }^{3}$ C. L. Lee, Y. C. Huang, L. C. Kuo, J. C. Oung, and F. C. Wu, Nanotechnology 17, 2390 (2006).

${ }^{4}$ A. S. Zuruzi, A. Kolmakov, N. C. MacDonald, and M. Moskovits, Appl. Phys. Lett. 88, 102904 (2006).

${ }^{5}$ H. Y. Xu, X. L. Liu, M. Li, Z. Chen, D. L. Cui, M. H. Jiang, X. P. Meng, L. L. Yu, and C. J. Wang, Mater. Lett. 59, 1962 (2005).

${ }^{6}$ M. A. Biot, J. Appl. Phys. 33, 1482 (1962); J. Acoust. Soc. Am.
34, 1254 (1962); M. A. Biot and D. G. Willis, ASME Trans. J. Appl. Mech. 24, 594 (1957).

${ }^{7}$ T. J. Plona, in Ultrasonics Symposium, 1982 (IEEE, New York, 1982), pp. 1044-1048.

${ }^{8}$ L. Ye, J. Liu, P. Sheng, and D. A. Weitz, Phys. Rev. E 48, 2805 (1993).

${ }^{9}$ S. Higashitani, T. Ichikawa, M. Yamamoto, M. Miura, and K. Nagai, Physica B 329-333, 299 (2003).

${ }^{10}$ N. P. Chotiros, J. Acoust. Soc. Am. 97, 199 (1995); 112, 1853 (2002).

${ }^{11}$ J. Ohkawa, J. Acoust. Soc. Am. 119, 709 (2006). 
${ }^{12}$ See, e.g., R. G. Yang, G. Chen, and M. S. Dresselhaus, Phys. Rev. B 72, 125418 (2005).

${ }^{13}$ D. O. Riese and G. H. Wegdam, Phys. Rev. Lett. 82, 1676 (1999).

${ }^{14}$ R. S. Penciu, G. Fytas, E. N. Economou, W. Steffen, and S. N. Yannopoulos, Phys. Rev. Lett. 85, 4622 (2000).

${ }^{15}$ R. Annamalai, J. D. West, A. Luscher, and V. V. Subramaniam, J. Appl. Phys. 98, 114307 (2005).

${ }^{16}$ S. Fujii, S. Honda, H. Machida, H. Kawai, K. Ishida, M. Katayama, F. Furuta, T. Hirao, and K. Oura, Appl. Phys. Lett. 90, 153108 (2007); Y. Wei, D. Weng, Y. C. Yang, X. B. Xhang, K. L. Jiang, L. Liu, and S. S. Fan, ibid. 89, 063101 (2006); Y. Wei, L. Xiao, F. Zhu, L. Liu, J. Tang, P. Liu, and S. S. Fan, Nanotechnology 18, 325702 (2007).

${ }^{17}$ C. Liu, Y. Tong, H. M. Cheng, D. Goldberg, and Y. Bando, Appl. Phys. Lett. 86, 223114 (2005).

${ }^{18} \mathrm{~J}$. Elich, Proceedings of the AIAA International Energy Conversion Conference, Portsmouth, Virginia, 2003 (unpublished).

${ }^{19}$ T.-I. Jeon, K. J. Kim, C. Kang, S. J. Oh, J. H. Son, K. H. An, D. J. Bae, and Y. H. Lee, Appl. Phys. Lett. 80, 3403 (2002).

${ }^{20} \mathrm{~T}$. de los Arcos, P. Oelhafen, and D. Mathys, Nanotechnology
18, 265706 (2007).

${ }^{21}$ See, e.g., F. P. Incropera, D. P. DeWitt, T. L. Bergman, and A. S. Lavine, Introduction to Heat Transfer (Wiley, New York, 2007), pp. 527-529.

${ }^{22}$ J. G. Berryman, J. Appl. Phys. 59, 1136 (1986).

${ }^{23}$ X. Jing, P. Sheng, and M. Zhou, Phys. Rev. Lett. 66, 1240 (1991).

${ }^{24}$ H. A. Makse, N. Gland, D. L. Johnson, and L. M. Schwartz, Phys. Rev. Lett. 83, 5070 (1999).

${ }^{25}$ Y.-Y. Chen and Z. Ye, Phys. Rev. E 64, 036616 (2001).

${ }^{26}$ M. S. Kushwaha, Int. J. Mod. Phys. B 10, 977 (1996).

${ }^{27}$ D. Stroud and F. P. Pan, Phys. Rev. B 17, 1602 (1978).

${ }^{28}$ L. Sanchis, A. Håkansson, F. Cervera, and J. Sánchez-Dehesa, Phys. Rev. B 67, 035422 (2003).

${ }^{29}$ See, e.g., D. J. Bergman and D. Stroud, Solid State Phys. 46, 147 (1992).

${ }^{30}$ D. L. Johnson and T. J. Plona, J. Acoust. Soc. Am. 72, 556 (1982).

${ }^{31}$ The densities of SWCNT and DMF are comparable. See, e.g., P. G. Collins and P. Avouris, Nanotubes for Electronics (Scientific American, 2000), Vol. 283, pp. 62-69. 